\title{
Phase domain boundary motion and memristance in gradient-doped FeRh nanopillars induced by spin injection: Supplementary Information
}

\author{
Rowan C. Temple, ${ }^{1}$ Mark C. Rosamond, ${ }^{2}$ Jamie R. Massey, ${ }^{1}$ Trevor P. Almeida, ${ }^{3}$ Edmund H. Linfield, ${ }^{2}$ \\ Damien McGrouther, ${ }^{3}$ Stephen McVitie, ${ }^{3}$ Thomas A. Moore, ${ }^{1}$ and Christopher H. Marrows ${ }^{1}$ a) \\ 1) School of Physics and Astronomy, University of Leeds, Leeds LS2 9JT, UK \\ ${ }^{2)}$ School of Electronic and Electrical Engineering, University of Leeds, Leeds LS2 9JT, \\ $U K$ \\ ${ }^{3)}$ SUPA, School of Physics and Astronomy, University of Glasgow, Glasgow G12 8QQ, \\ $U K$
}

(Dated: 25 January 2021)

\section{METHODS}

In this section we give further details of the experimental methods used in this work.

\section{A. Growth and characterisation}

The doped FeRh film was grown by DC sputtering at $600^{\circ} \mathrm{C}$ onto commercially obtained $\mathrm{MgO}(001)$ substrates ${ }^{1}$. The stack sequence is $\mathrm{MgO} / \mathrm{NiAl}(70 \mathrm{~nm}) / \mathrm{FeRh}(\mathrm{Pd}, \mathrm{Ir})(50 \mathrm{~nm})$ where the NiAl layer is an epitaxially matched metal used for the bottom contact to the nanopillar ${ }^{2}$. The doped FeRh layer was sputtered from a Pd and an Ir doped FeRh target. Angled magnetrons were used to allow rapid switching between target material growth and appropriate Pd or Ir densities were achieved by multiple $1 \mathrm{~nm}$ layer growths, each layer consisting of the appropriate ratio of $\mathrm{Pd}$ to $\mathrm{Ir}$ doping. A final anneal at $700^{\circ} \mathrm{C}$ for $1 \mathrm{hr}$ in vacuum allowed the FeRh to obtain the proper crystal structure and smooth out the doping profile within the film thickness. The film examined here is $\mathrm{NiAl}(70 \mathrm{~nm}) / \mathrm{Fe}_{50} \mathrm{Rh}_{47.2} \mathrm{Pd}_{2.8}(25 \mathrm{~nm}) /$ $\mathrm{Fe}_{50} \mathrm{Rh}_{47.1} \mathrm{Pd}_{2.2} \mathrm{Ir}_{0.7}(15 \mathrm{~nm}) / \mathrm{Fe}_{50} \mathrm{Rh}_{46.8} \mathrm{Pd}_{1.7} \mathrm{Ir}_{1.5}(10 \mathrm{~nm})$.

In Fig. S1 we show an X-ray diffraction scan of an unpatterned gradient-doped FeRh film grown in the same way as the one from which we fabricated our pillars. Bragg peaks are clearly visible for the (001) reflections of both the NiAl buffer and FeRh film, confirming that B2-ordering is present in both layers. The peaks for the FeRh are somewhat broadened due to the doping gradient causing a gradient in lattice constant. Analysis of the ratio of integrated intensities under the two peaks for the FeRh is consistent with a B2 chemical order parameter $S \sim 0.9$, a typical level for a thin film of this material $^{13}$.

\section{B. Patterning}

The bottom electrodes and nanopillars were defined using e-beam lithography on a JEOL JBX-6300FS system. Ar ion

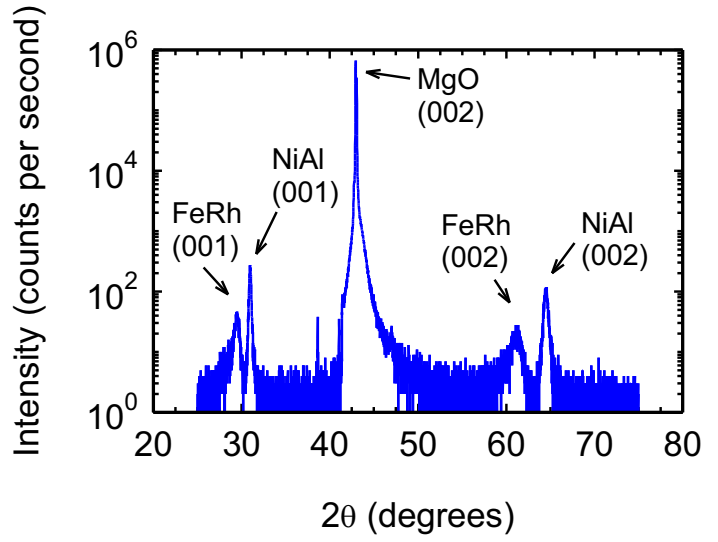

FIG. S1. X-ray diffraction scan of a gradient-doped FeRh film, with $\mathrm{MgO}$ substrate, NiAl buffer and FeRh film peaks marked and indexed.

milling through a ma-N2403 resist mask was used for pattern transfer ${ }^{4}$. The flying bridge contacts were created in a PMMA/MMA based lift-off process. An electron dose profile across the bridge used the contrast shift between the PMMA and MMA layers to define contact and flyover regions. The bridges contacts were sputter deposited $\mathrm{Ti}(6 \mathrm{~nm}) / \mathrm{Au}(300 \mathrm{~nm})$. An insert PMGI resist layer was used to create undercut so as to improve lift-off.

\section{Measurements}

The HAADF and EDS imaging and analysis shown in Fig. 1c of the main text were carried out on JEOL Atomic Resolution Microscope (JEM-ARM200F) TEM, operating at $200 \mathrm{kV}$. The elemental data was acquired with a Bruker XFlash EDS detector.

Transport measurements were performed in a liquid nitrogen cooled Oxford Instruments OptistatDN with a temperature range of 77-500 K. A Keithley 6221 current source was used for pulsed current injection and signals were detected using a Keithley 2182 nanovoltmeter in the quasi-four point geometry shown in Fig. 1b of the main text. All measurements were carried out at zero applied field.

\footnotetext{
${ }^{a)}$ Electronic mail: c.h.marrows@ @leeds.ac.uk
} 


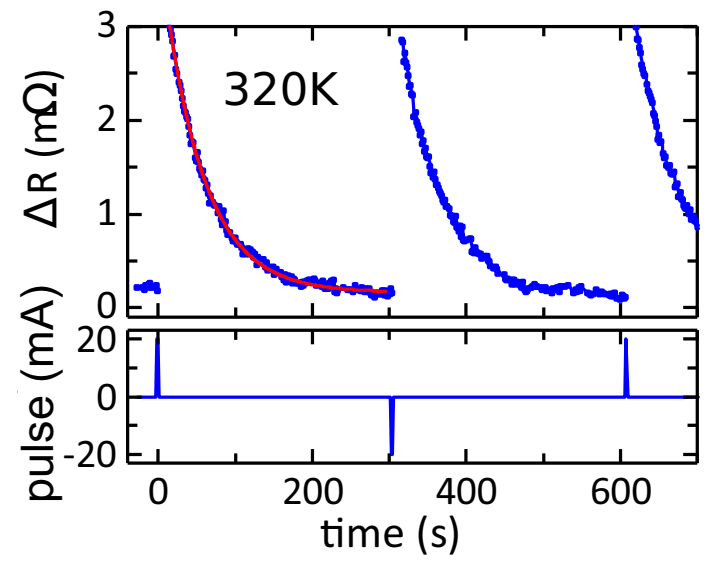

FIG. S2. Heating due to current pulses. The top panel shows resistance change response to current pulse trains through the $300 \mathrm{~nm}$ diameter nanopillar at $320 \mathrm{~K}$. The pulse train times and current amplitude are shown in the bottom panel. Each pulse train consists of $10^{6}$ individual pulses each lasting $1 \mu \mathrm{s}$, delivered with a $10 \%$ duty cycle. This allows a $9 \mu$ s cooling period after each pulse, which proved necessary to increase the total charge delivered while managing the heating effects of the current. The pulse response is symmetric with positive and negative currents as expected for Joule heating effects. The red line shows an exponential decay fit to the post-pulse cooling. The fitting parameters give an equivalent heating amplitude of $1.4 \mathrm{~K}$ and a decay time of $54 \mathrm{~s}$.

\section{CURRENT-DRIVEN HEATING}

A side-effect of high current densities in nanoscale devices is Joule heating, which will affect the device resistance. In Fig. S2 here, we show the effect of applying a pulse train of $10^{6}$ pulses, each of $1 \mu$ s duration, with a duty cycle of $10 \%$ to the $300 \mathrm{~nm}$ nanopillar device. The amplitude of each pulse was $20 \mathrm{~mA}$, corresponding to a current density of $\sim 3 \times 10^{11} \mathrm{~A} / \mathrm{m}^{2}$. This experiment was carried out at $320 \mathrm{~K}$, a temperature at which the nanopillar is fully $\mathrm{AF}$ and no phase boundary is present, as can be seen in Fig. 3 in the main text.

The measured resistance of the nanopillar rises by $\Delta R \approx$ $3 \mathrm{~m} \Omega$ at the time of the pulse before exponentially relaxing back to its equilibrium value on a $1 / e$ timescale of $54 \mathrm{~s}$. By making a comparison with the linear regime in Fig. 2 of the main text, we determined that this resistance rise corresponds to a temperature rise of about $1.4 \mathrm{~K}$. The response is the same regardless of the direction in which the current pulses flow, showing that in this case the resistance change is entirely due to Joule heating.

Test nanopillars were stable at this duty cycle, but often failed when the $9 \mu$ sap between pulses was reduced much below this value. This indicates that the nanopillar temperature briefly rises well above this value but cools on a $\mu$ s timescale, fast enough to be beyond the time resolution of these measurements. The principal heatsink for cooling will be the region of substrate just under the pillar, and we attribute this temperature rise and slow decay to the heating of that region and gradual cooling as heat is conducted away into the rest of the substrate.

\section{REFERENCES}

${ }^{1}$ C. Le Graët, M. A. de Vries, M. McLaren, R. M. Brydson, M. Loving, D. Heiman, L. H. Lewis, and C. H. Marrows, "Sputter Growth and Characterization of Metamagnetic B2-ordered FeRh Epilayers," J. Vis. Exp. , e50603 (2013).

${ }^{2}$ D. Kande, S. Pisana, D. Weller, D. E. Laughlin, and J. G. Zhu, "Enhanced B2 ordering of FeRh thin films using B2 NiAl underlayers," IEEE Trans. Magn. 47, 3296-3299 (2011).

${ }^{3}$ M. A. de Vries, M. Loving, A. P. Mihai, L. H. Lewis, D. Heiman, and C. H. Marrows, "Hall-effect characterization of the metamagnetic transition in FeRh," New J. Phys. 15, 013008 (2013).

${ }^{4}$ R. C. Temple, T. P. Almeida, J. R. Massey, K. Fallon, R. Lamb, S. A. Morley, F. Maccherozzi, S. S. Dhesi, D. McGrouther, S. McVitie, T. A. Moore, and C. H. Marrows, "Antiferromagnetic-ferromagnetic phase domain development in nanopatterned FeRh islands," Phys. Rev. Materials 2, 104406 (2018). 